\title{
Correction to: Developing Nucleon Self-Energies to Generate the Ingredients for the Description of Nuclear Reactions
}

Correction to:

M. C. Atkinson, Developing Nucleon Self-Energies

to Generate the Ingredients for the Description

of Nuclear Reactions, Springer Theses, https://doi.org/10.1007/978-3-030-53114-0

The spelling of the book title was incorrect. Now, the book title has been corrected as "Developing Nucleon Self-Energies to Generate the Ingredients for the Description of Nuclear Reactions". 